\title{
Thresholds of Lasing as Solutions of Characteristic Equation for a VCSEL-type Layered Structure
}

\author{
Volodymyr O. Byelobrov, Alexander I. Nosich \\ Institute of Radiophysics and Electronics NASU, ul. Proskury 12, Kharkiv 61085, Ukraine \\ E-mail: volodia.byelobrov@gmail.com
}

\begin{abstract}
Thresholds of lasing for a cavity with a quantum well placed between two distributed Bragg reflectors are investigated as a specific eigenvalue problem with "active" imaginary part of the quantum well refractive index. The characteristic equation is obtained from the transmission-matrix formalism. Preliminary results show that the frequencies of lasing are determined mainly by the size and material of the cavity, and the thresholds are sensitive to the quantum-well size and location and can be lowered by the proper tuning of the Bragg reflectors.
\end{abstract}

Keywords: transmission matrix, laser, eigenproblem, VCSEL.

\section{INTRODUCTION}

In today's photonics, active-material layers commonly called quantum wells (QWs) and embedded in epitaxially grown semiconductor microcavities are widely used. This relates first of all to the vertical cavity surfaceemitting lasers (VCSELs) and also to laser-emitting diodes, where cascading the QWs enables to shift the emission wavelength to the lower frequencies, etc. [1-3].

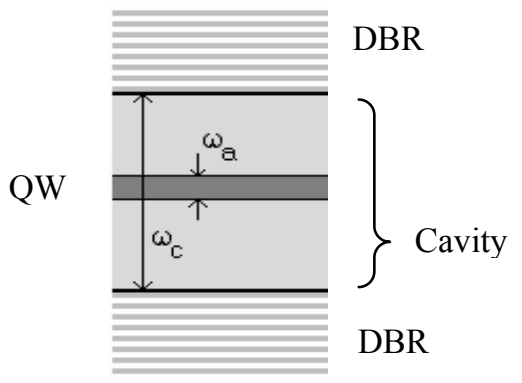

Fig. 1. Geometry of the VCSEL-type layered structure.

In a semiconductor microcavity, a high intensity optical field is usually confined by using distributed Bragg reflectors (DBRs) composed of the quarter-wavelength multilayers grouped in periodic pairs. VCSEL-type geometry is depicted (not in scale) in Fig. 1. Within the past years, much attention has been concentrated on making use of the microcavity effect in semiconductor optical devices to improve their performance in several respects. It has been noticed that the lasing wavelength for a VCSEL is determined mainly by the optical properties of the mirrors and the cavity. The threshold current for the onset of lasing, however, is known to be sensitive mainly to the shape and placement of the current-injection electrodes. An understanding of the relationship between the QW location, composition, and width and the cavity natural-mode field is important in order to achieve low room-temperature thresholds. However, direct analysis of the dependence of thresholds on the microcavity and DBRs geometry has largely escaped attention of researchers and has been usually substituted with the analysis of Q-factors.

\section{TRANSMISSION MATRIX}

Consider an auxiliary problem about the transmission and reflection of the plane wave normally incident on arbitrary multilayer dielectric structure located in free space as shown in Fig. 2. The matrix of the transformation of the complex amplitudes of the waves on the first boundary of layers, $t$, can be introduced as

$$
\left(\begin{array}{l}
a_{0} \\
b_{0}
\end{array}\right)=t\left(k y_{1}, \varepsilon_{0}, \varepsilon_{1}\right)\left(\begin{array}{l}
a_{1} \\
b_{1}
\end{array}\right)
$$




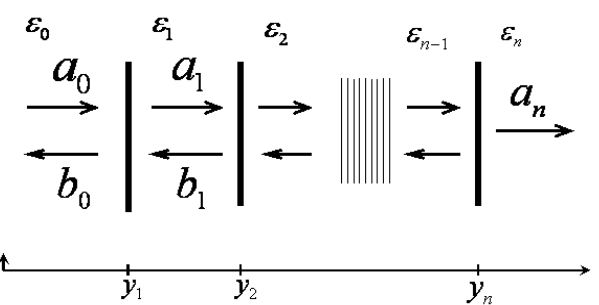

Fig. 2. Sketch of the auxiliary multilayer scattering problem (right).

A general expression for the transmission matrix is

$$
t(\kappa, \alpha, \beta)=\frac{1}{2}\left(\begin{array}{ll}
e^{i(\alpha-\beta) \kappa}(1+\alpha / \beta) & e^{i(\alpha+\beta) \kappa}(1-\alpha / \beta) \\
e^{-i(\alpha+\beta) \kappa}(1-\alpha / \beta) & e^{-i(\alpha-\beta) \kappa}(1+\alpha / \beta)
\end{array}\right)
$$

This matrix possesses the following properties, which have clear physical meaning:

$$
\begin{array}{lll}
\text { (i) } & t(\kappa, \alpha, \beta) \cdot t(\kappa, \beta, \alpha)=I, & \text { (ii) } \quad t(\kappa, \alpha, \alpha)=I, \quad \text { (iii) } \quad t^{-1}(\kappa, \alpha, \beta)=t(\kappa, \beta, \alpha), \\
\text { (iv) } & t(\kappa, \alpha, \beta) \cdot t(\kappa, \beta, \gamma)=t(\kappa, \alpha, \gamma), & (\mathrm{v}) \quad \operatorname{det} t(\kappa, \alpha, \beta)=\beta / \alpha .
\end{array}
$$

The transformation matrix for the whole layered structure can by obtained as a product of partial transmission matrices, $t\left(\kappa_{i}, \varepsilon_{i-1}, \varepsilon_{i}\right)$, where $\kappa_{i}=k y_{i}$, namely

$$
P=\left(\begin{array}{ll}
p_{11} & p_{12} \\
p_{21} & p_{22}
\end{array}\right)=t\left(\kappa_{1}, \alpha_{1}, \beta_{1}\right) \cdot t\left(\kappa_{2}, \alpha_{2}, \beta_{2}\right) \cdot \ldots \cdot t\left(\kappa_{n}, \alpha_{n}, \beta_{n}\right)
$$

This is the matrix linking together the waves outside of the layered structure as

$$
\left(\begin{array}{l}
a_{0} \\
b_{0}
\end{array}\right)=P\left(\begin{array}{c}
a_{n} \\
0
\end{array}\right)
$$

The reflection coefficient is the ratio of the complex amplitudes of the outgoing (i.e., reflected) and the incoming (i.e., incident) waves in the 0 -th domain, and the transmission coefficient is the ratio of the outgoing (i.e., transmitted) wave after the layered structure in the $n$-th domain and incoming (i.e., incident) one before it. Therefore we obtain from (4) that

$$
R=b_{0} / a_{0}=p_{21} / p_{11}, T=a_{n} / a_{0}=1 / p_{11}
$$

\section{CHARACTERISTIC EQUATION}

For the solution of an eigenproblem we may use the boundary conditions to form a set of linear equations where unknowns are complex amplitudes of electromagnetic waves inside and outside of the structure. This system size is $2 n \times 2 n$, where $n$ is the number of boundaries. Then we can obtain an analytical representation of the reflection and transmission coefficients, each of which is a ratio of two expressions with a common denominator. Using (5) we can obtain it in a simpler way, namely

$$
p_{11}=0
$$

From the other hand, when solving the eigenproblem for the layered structure we should consider only two waves outgoing from the structure (no incoming one). This situation is characterized by the equation differing from (4) by a zero instead of $a_{0}$, so that simultaneously $0=p_{11} a_{n}$ and $b_{0}=p_{21} a_{n}$, which obviously leads to (6).

\section{MODELLING OF DBR MICROCAVITY LASER}

Based on the above formalism, we have modelled the lasing spectra and associated thresholds of the modes in a laterally uniform model of VCSEL-type semiconductor layered structure depicted in Fig. 1. It consists of the cavity whose width is $w_{c}$, sandwiched between two DBRs made of $N_{T}$ and $N_{B}$ pairs of high and low-index layers whose thicknesses are $w_{H}$ and $w_{L}$, respectively. Inside the cavity there is a $\mathrm{QW}$ of the width $w_{a}$. Mathematically, the lasing modes of a VCSEL can be viewed as source-free solutions to the lasing eigenvalue 
problem (LEP) for the Maxwell equations, introduced in [4]. We look for the non-attenuating field $\sim e^{-i k c t}$, where $k=\operatorname{Re} k>0$ and $c$ is the light velocity.
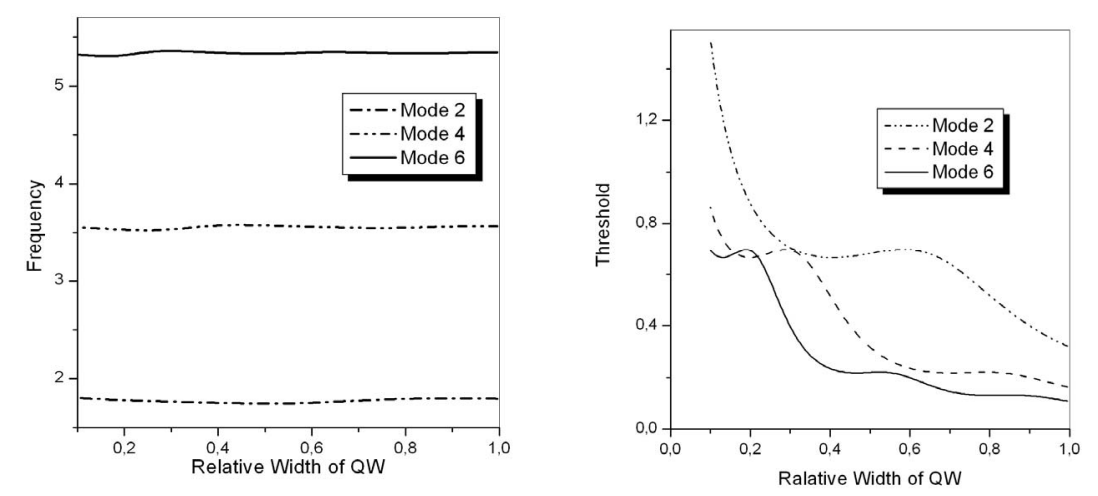

Fig. 3. Dependences of even-mode frequencies (left) and thresholds (right) on the relative width of the centered $Q W$ for the GaAs cavity in free space. Odd modes display similar behaviour.

We assume that the electromagnetic field does not vary along the axes $x$ and $z$, and can be characterized by one scalar function $U$, which represents either $E_{z}$ or $H_{z}$ component depending on the polarization. Off the layer interfaces, this function must satisfy the Helmholtz equation, $\left[\Delta+k^{2} v^{2}(y)\right] U(y)=0$. Here, step-like function $v(y)$ is assumed 1 outside the cavity and DBRs, i.e. in the lower and upper half-spaces filled with air; inside the layered structure it takes the values corresponding to the refractive indices of semiconductor cavity, $\alpha_{c}$, and the high and low-index materials of the DBR pairs, $\alpha_{H}$ and $\alpha_{L}$. All $\alpha_{s}(s=c, H, L)$ are assumed real or complexvalued with positive imaginary parts, i.e. may display absorption. The parameter $v=\operatorname{Re} \alpha_{c}-i \gamma$ is assumed, however, complex-valued with a negative imaginary part inside the QW region that corresponds to the active (or, equivalently, negative-loss) material. The optical field tangential components must satisfy the continuity conditions at the layer interfaces including the boundary between QW and the rest of cavity, and obey the outgoing-wave radiation condition at infinity $(|y| \rightarrow \infty)$.

Keeping in mind source-free solutions to this boundary-value problem, we seek its eigenvalues as a discrete set of pairs of real-valued parameters, $\left(\kappa_{n}, \gamma_{n}\right)$. The first of them is the normalized frequency of lasing, $\kappa=k w_{c}$ and the second is the associated threshold material gain. Note that the gain per unit length, frequently met in the Fabry-Perot cavities descriptions, is obtained as $g=k \gamma$. This formulation is different from the "classical" formulation of the eigenvalue problem for an open cavity with the complex-valued frequency $k$ being eigenvalue parameter. Note that LEP is still a linear problem, however takes into account the presence of the active region and enables one to extract the thresholds, in terms of $\gamma$, in addition to the natural-mode wavelengths. This is more adequate to the lasing than determining the Q-factors $\left(Q_{s}=\left|\operatorname{Re} k_{s} / 2 \operatorname{Im} k_{s}\right|\right)$ and can be qualified as building a 1-D "warm" model of VCSEL - see [5].

At first, we computed the dependences of the lasing frequencies and thresholds, in the E-polarisation case, on the relative width, $w_{a} / w_{c}$, of the QW placed in the centre of the GaAs cavity $\left(\alpha_{c}=3.53\right)$ without DBRs, i.e. immersed into air - see Figs. 3. For such a structure, solutions of the LEP corresponding to the modes $E_{n}$ asymptotically behave as $\gamma_{n}=\left[\kappa_{n}\left(w_{a} / w_{c}\right)\right]^{-1} \ln \left[\left(\alpha_{c}+1\right) /\left(\alpha_{c}-1\right)\right]$ and $\kappa_{n}=\pi n / \alpha_{c}$, for $n \gg 1$. The oscillations on the curves of thresholds in Fig. 3 correspond to the better or worth overlap between the active layer and the $\mathrm{E}_{n}$ mode electric-field pattern. 


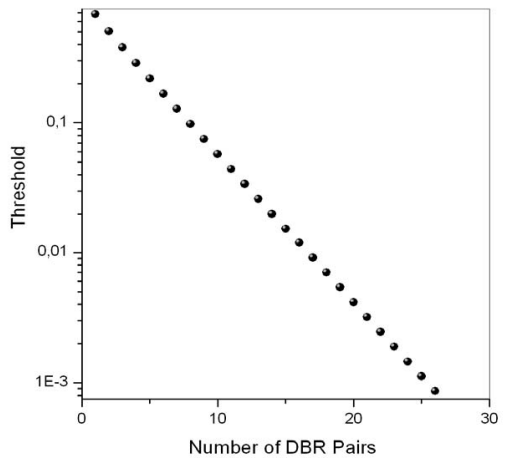

Fig. 4. Dependence of threshold on the number of DBR pairs, mode $E_{10}$.

Fig. 4 shows the values of the threshold of the mode $\mathrm{E}_{10}$ in the GaAs cavity with $\alpha_{c}=3.53$ and a centred QW with $w_{a}=0.1 w_{c}$ sandwiched between two identical DBRs. Each reflector consists of $N$ pairs of GaAs/AlGaAs layers $\left(\alpha_{H}=3.53, \alpha_{L}=3.08\right)$ whose widths are $w_{H} / w_{c}=0.2473$ and $w_{L} / w_{c}=0.2834$, respectively. As one can see the adding of each new pair essentially decreases the threshold.

\section{CONCLUSIONS}

We have presented some preliminary results of the accurate numerical analysis of the lasing characteristics of the 1-D model of a VCSEL equipped with QW layer and two DBRs. They demonstrate that the modal thresholds are sensitive to the width and location of QW inside the cavity and to the reflectivities of DBRs, while the lasing frequency depends mainly on the cavity width and modal index. This analysis opens new ways of manipulation and tailoring of the modal thresholds in epitaxially grown layered structures with active regions.

\section{REFERENCES}

[1] G. M. Yang, et al., Microcavity effects on the spontaneous emission from InGaAdGaAs quantum wells, J. Appl. Phys., vol. 78, no. 6, pp. 3605-3609, 1995.

[2] I.-S. Chung, et al., Effect of outermost layers on resonant cavity enhanced devices, J. Appl. Phys., vol. 96, no. 5, pp. 2423-2427, 2004.

[3] X. H. Zhang, et al., Crack-free fully epitaxial nitride microcavity with AlGaN/GaN distributed Bragg reflectors and InGaN/GaN quantum wells, Appl. Phys. Lett., vol. 88, pp. 191111, 2006.

[4] E.I. Smotrova, A.I. Nosich, Mathematical analysis of the lasing eigenvalue problem for the WG modes in a 2-D circular microcavity, Optical and Quantum Electronics, vol. 36, no. 1-3, pp. 213-221, 2004.

[5] E.I. Smotrova, A.I. Nosich, T.M. Benson, P. Sewell, Cold-cavity thresholds of microdisks with uniform and non-uniform gain: quasi-3D modelling with accurate 2D analysis, IEEE J. Selected Topics Quantum Electronics, vol. 11, no. 5, pp. 1135-1142, 2005. 